\title{
A study on correction equations for the effect of seafloor topography on ocean bottom magnetotelluric data
}

\author{
Tetsuo Matsuno ${ }^{1}$, Nobukazu Seama ${ }^{1,2,3}$, and Kiyoshi Baba ${ }^{4}$ \\ ${ }^{1}$ Graduate School of Science and Technology, Kobe University, Kobe 657-8501, Japan \\ ${ }^{2}$ Research Center for Inland Seas, Kobe University, Kobe 657-8501, Japan \\ ${ }^{3}$ IFREE, Japan Agency for Marine-Earth Science and Technology, Kanagawa 237-0061, Japan \\ ${ }^{4}$ Earthquake Research Institute, University of Tokyo, Tokyo 113-0032, Japan
}

(Received June 17, 2005; Revised April 15, 2007; Accepted May 10, 2007; Online published August 31, 2007)

\begin{abstract}
Consideration of the effect of seafloor topography on ocean bottom magnetotelluric data is needed to estimate a reliable resistivity structure. Selection of the correction equation which precisely takes account of distortions is important when we correct the effect of seafloor topography with forward modeling. Corrections of synthetic data based on three different correction equations are carried out, and the corrected responses and the true response are compared to investigate which correction equation is the best in application to distorted magnetotelluric data on the seafloor. Differences in the corrected responses are remarkable at periods shorter than several thousands seconds. These differences are caused by differences in treatment of the distortions of magnetic field and in robustness of the correction equation to a resistivity structure assumed for the topographic correction. The results suggest that the correction equation of Nolasco et al. (1998) should be used because it better accommodates the estimation of unknown resistivity structures. We apply the correction equation of Nolasco et al. (1998) to a response observed in the Mariana area. The one-dimensional resistivity structure estimated from the corrected response explains almost all the components of the observed response.
\end{abstract}

Key words: Marine magnetotellurics, seafloor topographic effect, correction of distortions.

\section{Introduction}

Correcting the effect of seafloor topography on ocean bottom magnetotelluric data is essential to estimate a reliable resistivity structure. Since seafloor topography is an undulating interface between conductive seawater and resistive crust, it generates anomalous flows of electric currents in seawater and anomalous magnetic fields. These anomalous electric and magnetic fields will contaminate observed data, therefore a resistivity structure could be wrongly estimated when the contaminated data is used (e.g., Baba and Chave, 2005).

The effect of seafloor topography has been taken into account by two different methods. (1) Correcting an observed impedance tensor based on a distortion equation which relates a distorted impedance tensor to an undistorted impedance tensor, and then estimating a resistivity structure from the corrected impedance tensor (Correction method). The correction method is further classified into approaches with or without forward modeling. For the approach with forward modeling, the electromagnetic fields are modeled for a resistivity structure with and without topography to acquire distortion terms which are used for the correction (e.g., Nolasco et al., 1998). On the other hand, for the approach without forward modeling, tensor decomposition is frequently used because of its availability, es-

Copyright (c) The Society of Geomagnetism and Earth, Planetary and Space Sciences (SGEPSS); The Seismological Society of Japan; The Volcanological Society of Japan; The Geodetic Society of Japan; The Japanese Society for Planetary Sciences; TERRAPUB pecially on land data (e.g., Bahr, 1988; Groom and Bailey, 1989). However, the tensor decomposition will often fail in application to seafloor data if only galvanic distortion is assumed (Nolasco et al., 1998). (2) Estimating a resistivity structure through an inversion directly incorporating seafloor topography (e.g., Goto et al., 2003; Constable and Heinson, 2004) (Direct method). The correction method with forward modeling and the direct method take account of seafloor topography explicitly. However, the direct method needs a three-dimensional (3-D) inversion incorporating 3-D seafloor topography which is expensive to perform, and it is not efficient when a subsurface structure is expected to be 1-D or 2-D. Thus, the correction method with forward modeling is the most reasonable method to treat the effect of seafloor topography at the present stage.

In this study, we investigate the correction method with forward modeling. Selection of the correction equation which precisely takes account of distortions is essential when we correct the effect of seafloor topography with forward modeling. First, we introduce three different equations for the topographic correction. Then, we test these correction equations for a synthetic magnetotelluric response generated from 3-D topography over a 1-D structure to investigate which correction equation produces the true 1-D response without seafloor topography. Furthermore, we apply the topographic correction to real data observed in the Mariana area and estimate a resistivity structure from the corrected impedance tensor. Finally, we examine reliability of the estimated resistivity structure, and show the 
effectiveness of estimating a resistivity structure by inverting a corrected impedance tensor.

\section{Three Topographic Correction Equations}

We classify topographic correction equations from previous work into the following three equations. The difference in the three topographic correction equations is in representing distortions of the electric and magnetic fields. Equations (1) representing the horizontal electric and magnetic fields distorted by seafloor topography $(\mathbf{E}$ and $\mathbf{B})$ are

$$
\begin{aligned}
& \mathbf{E}=\mathbf{E}_{m}+\mathbf{M E}, \\
& \mathbf{B}=\mathbf{B}_{m}+\mathbf{K}_{e} \mathbf{E},
\end{aligned}
$$

and the correction equation is

$$
\mathbf{Z}_{c}=(\mathbf{I}-\mathbf{M}) \mathbf{Z}_{o}\left(\mathbf{I}-\mathbf{K}_{e} \mathbf{Z}_{o}\right)^{-1},
$$

where $\mathbf{E}_{m}$ and $\mathbf{B}_{m}$ are the horizontal electric and magnetic fields without the topographic effects, $\mathbf{M}$ and $\mathbf{K}_{e}$ are $2 \times 2$ complex tensors to represent the electric and magnetic topographic effects, respectively, including both galvanic and inductive distortions, $\mathbf{I}$ is the identity tensor, $\mathbf{Z}_{o}$ is an observed impedance tensor, and $\mathbf{Z}_{c}$ is a corrected impedance tensor. These equations are identical to those of Nolasco et al. (1998) which are introduced by approximating the integral equations based on scattering theory to represent distortions of electric and magnetic fields.

Equations (2) are

$$
\begin{aligned}
& \mathbf{E}=\mathbf{E}_{m}+\mathbf{M E}, \\
& \mathbf{B}=\mathbf{B}_{m}+\mathbf{K}_{b} \mathbf{B},
\end{aligned}
$$

and the correction equation is

$$
\mathbf{Z}_{c}=(\mathbf{I}-\mathbf{M}) \mathbf{Z}_{o}\left(\mathbf{I}-\mathbf{K}_{b}\right)^{-1}
$$

where $\mathbf{K}_{b}$ is a $2 \times 2$ complex tensor to represent the magnetic topographic effect. These equations are based on Schmucker (1970), Jiracek et al. (1989), and Jiracek (1990), which are applied to land topographic corrections. They assumed that distorted electric and magnetic fields were combined with undistorted electric and magnetic fields through topographic effect terms $\mathbf{C}$ and $\mathbf{D}$,

$$
\begin{aligned}
& \mathbf{E}=\mathbf{C E}_{m}, \\
& \mathbf{B}=\mathbf{D B}_{m} .
\end{aligned}
$$

Schmucker (1970) introduced these equations from the quasi-stationary approximations of Maxwell's equations and confirmed their validity with the analysis of magnetograms. We divide the right-hand sides of Eqs. $\left(2 \mathrm{a}^{\prime}\right)$ and $\left(2 b^{\prime}\right)$ into the undistorted part and the distorted part as Eqs. (2a) and (2b). Although Eqs. $\left(2 a^{\prime}\right)$ and $\left(2 b^{\prime}\right)$ originally assumed that both electric and magnetic fields are three components and hence $\mathbf{C}$ and $\mathbf{D}$ are $3 \times 3$ tensors, we ignore the contribution of the vertical component.

Equation (3) assumes that an impedance tensor distorted by seafloor topography $\mathbf{Z}$ is represented as the product of a topographic effect tensor $\mathbf{Z}_{t}$ and an impedance tensor without topographic distortions $\mathbf{Z}_{m}$,

$$
\mathbf{Z}=\mathbf{Z}_{t} \mathbf{Z}_{m}
$$

The same relationship is assumed between an observed impedance tensor $\mathbf{Z}_{o}$ and a corrected impedance tensor $\mathbf{Z}_{c}$ through the topographic effect tensor,

$$
\mathbf{Z}_{o}=\mathbf{Z}_{t} \mathbf{Z}_{c}
$$

Rearranging (3a) and (3b), the correction equation is introduced as

$$
\mathbf{Z}_{c}=\mathbf{Z}_{m} \mathbf{Z}^{-1} \mathbf{Z}_{o}
$$

Heinson and Lilley (1993) and Baba and Chave (2005) used this correction equation.

The practical procedure for the correction is as follows. (1) $\mathbf{E}$ and $\mathbf{B}$, and $\mathbf{E}_{m}$ and $\mathbf{B}_{m}$ are calculated by using forward modeling with and without the inclusion of topographic change into the model, respectively. A resistivity structure needs to be assumed for these calculations because we do not know the true resistivity structure. (2) Topographic effect terms $\mathbf{M}$ and $\mathbf{K}_{b}$, or $\mathbf{Z}_{t}$ are calculated from $\mathbf{E}, \mathbf{B}, \mathbf{E}_{m}$, and $\mathbf{B}_{m}$, or $\mathbf{Z}$ and $\mathbf{Z}_{m}$ based on Eqs. (1a) and (1b), (2a) and (2b), or (3a). Note that $\mathbf{Z}$ is not the same as an observed impedance tensor $\mathbf{Z}_{o}$ unless the assumed resistivity structure is the same as the real resistivity structure. (3) $\mathbf{Z}_{o}$ is corrected by multiplying the topographic effect terms based on correction equations (1c), (2c), or (3c). Errors of the corrected tensor $\mathbf{Z}_{c}$ are calculated from those of $\mathbf{Z}_{o}$ following the error propagation law.

Flattening Surface 3-D modeling (FS3D) (Baba and Seama, 2002) is used for the forward modeling. FS3D is improved finite difference forward modeling of Mackie et al. (1994), which allows easy incorporation of rugged seafloor topography in computation. FS3D can solve problems pertaining to thin sheet modeling (e.g., Vasseur and Weidelt, 1977; Heinson and Lilley, 1993). For example, by using FS3D, we can calculate electromagnetic fields at short periods not realized by the thin sheet approximation. The computation accuracy of FS3D was guaranteed by comparing with thin sheet modeling (Baba and Seama, 2002), and with analytical solutions of Schwalenberg and Edwards (2004) (Baba and Chave, 2005).

\section{Synthetic Test on Three Topographic Correc- tion Equations}

We conducted a synthetic test on three topographic correction equations. A synthetic impedance tensor, which is calculated from known seafloor topography and resistivity structure, is corrected with each topographic correction equation. The synthetic impedance tensor at the center of the modeling area (Fig. 1(a)) generated by the forward modeling of 3-D topography over a 1-D resistivity structure (Fig. 1(b)) with the addition of $3 \%$ Gaussian noise. The calculation area is a square whose side length is $3200 \mathrm{~km}$. This area is discretized into numerical rectangular blocks whose horizontal dimensions are $1 \mathrm{~km}$ near the center of the model and become larger further from the center. The topographic change within each block is averaged for the data 
(a)

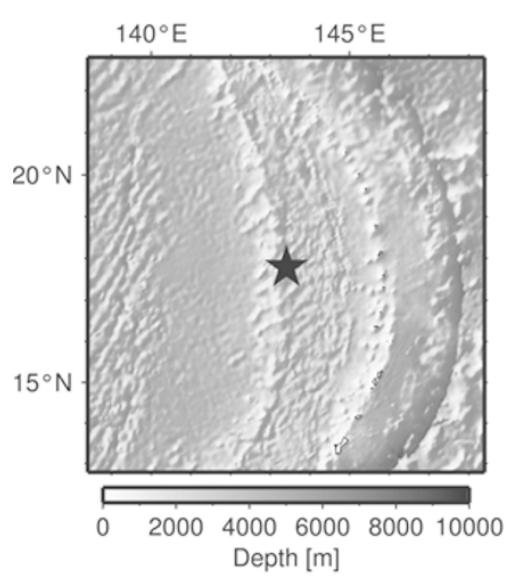

(b)

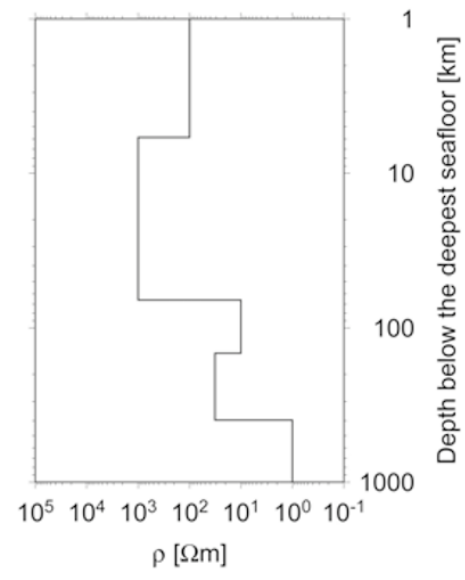

Fig. 1. (a) The seafloor topography map in the Mariana area. The star indicates the observation point for the synthetic and real data. (b) The 1-D resistivity structure model beneath the seafloor used to generate the synthetic data.

points. The seafloor topography data is given from a blend of Observed and Predicted relief data with grid spacing of 30 seconds (Geoware). The resistivity structure assumed for the calculation is a uniform half-space of $100 \mathrm{Ohm}-\mathrm{m}$. Nine periods used for the analysis are equally spaced in the logarithmic scale from 320 to 81,920 seconds.

The assessment of the result of the topographic correction is based on comparisons with the responses (apparent resistivities and phases) corrected and calculated from the model without the topographic change (flat-lying seafloor). The following features represent the good result of the topographic correction. The corrected apparent resistivity and phase (dots in Fig. 2) approach the flat-lying seafloor apparent resistivity and phase (solid lines in Fig. 2) for the off-diagonal components, and because the true resistivity structure is one-dimensional, the corrected apparent resistivity approach zero for the diagonal components. The result using Eq. (1) (Fig. 2(a)) shows these features, indicating that the effect of seafloor topography is well corrected. On the other hand, the results using Eqs. (2) (Fig. 2(b)) and (3) (Fig. 2(c)) do not show these features at periods shorter than several thousands seconds in all the components of apparent resistivity and in the xy component of the phase, indicating that the effect of seafloor topography is not well corrected at these periods. We evaluate the performance of each correction equation by using RMS misfit between the corrected impedance and the true 1-D impedance. The RMS misfit is defined as

$$
\begin{gathered}
\mathrm{RMS}=\sqrt{\frac{1}{2 N}} \times \\
\sqrt{\sum_{i=1}^{N}\left\{\left(\frac{\operatorname{Re}\left\{Z_{c}^{i}\right\}-\operatorname{Re}\left\{Z_{m}^{i}\right\}}{\delta Z_{c}}\right)^{2}+\left(\frac{\operatorname{Im}\left\{Z_{c}^{i}\right\}-\operatorname{Im}\left\{Z_{m}^{i}\right\}}{\delta Z_{c}}\right)^{2}\right\}}
\end{gathered}
$$

where $N$ is the number of data and $\delta Z_{c}$ is the standard error of a corrected impedance $Z_{c}$. RMS misfits with the correction Eqs. (1), (2), and (3) are 2.6, 6.9, and 6.7, respectively (Table 1). Thus, the topographic correction with Eq. (1) is the best.

The differences in the results of the synthetic test are
Table 1. Comparison of RMS misfits which are calculated by using each correction equation and by assuming uniform half-spaces of different resistivities.

\begin{tabular}{lccc}
\hline & 10 Ohm-m & 100 Ohm-m & 1000 Ohm-m \\
\hline Eq. (1) & 8.5 & 2.6 & 2.5 \\
Eq. (2) & 14.5 & 6.9 & 13.5 \\
Eq. (3) & 14.3 & 6.7 & 12.6 \\
\hline
\end{tabular}

explained by (1) differences in treatment of distortions of the magnetic field, and (2) differences in robustness of the correction equations to the resistivity structure assumed for the correction. The differences in the results are remarkable at periods shorter than several thousands seconds, and therefore reflect differences in treatment of the anomalous magnetic field among the three correction equations. This is because distortions of magnetic field are generally more significant at shorter periods while those of electric field prevail in all the analysis periods (e.g., Chave and Smith, 1994). In Eq. (1), the anomalous part of the magnetic field is represented as the matrix product of the electric field and the topographic effect term. On the other hand, in Eq. (2), the anomalous part of the magnetic field is represented as the matrix product of the magnetic field and the topographic effect term. In Eq. (3), we cannot identify the representation of the anomalous part of the magnetic field. These differences suggest that the effect of seafloor topography is primarily related to the anomalous horizontal electric current (electric field) induced by the rugged seafloor topography, and that the horizontal electric field represents the effect of seafloor topography better than the horizontal magnetic field.

The differences in robustness of the correction equations to the resistivity structure assumed for the correction become clear from transformations of the equations. Equation 1 approximates the theoretical integral equation for distortions (e.g., Hohmann, 1975; Nolasco et al., 1998),

$$
\mathbf{E}(\mathbf{r})=\mathbf{E}_{m}(\mathbf{r})-i \omega \mu_{0} \int_{V} \delta \frac{1}{\rho\left(\mathbf{r}^{\prime}\right)} \mathbf{G}\left(\mathbf{r}, \mathbf{r}^{\prime}\right) \mathbf{E}\left(\mathbf{r}^{\prime}\right) d \mathbf{r}^{\prime},
$$


(a)

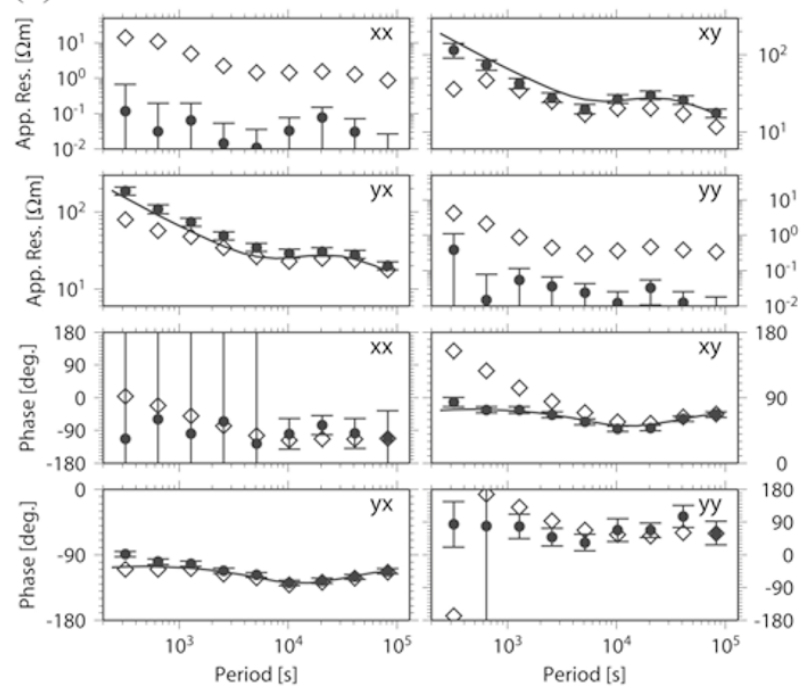

(b)
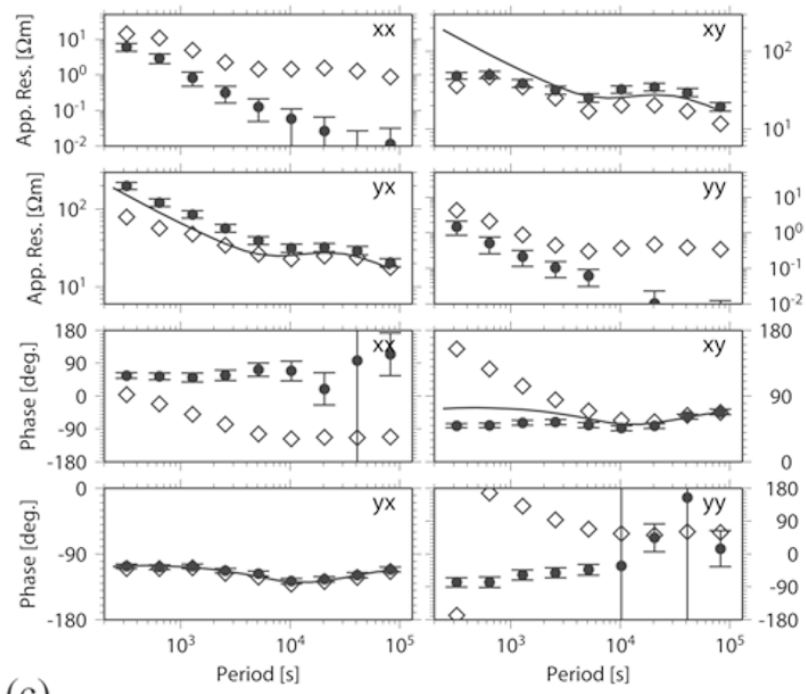

(c)
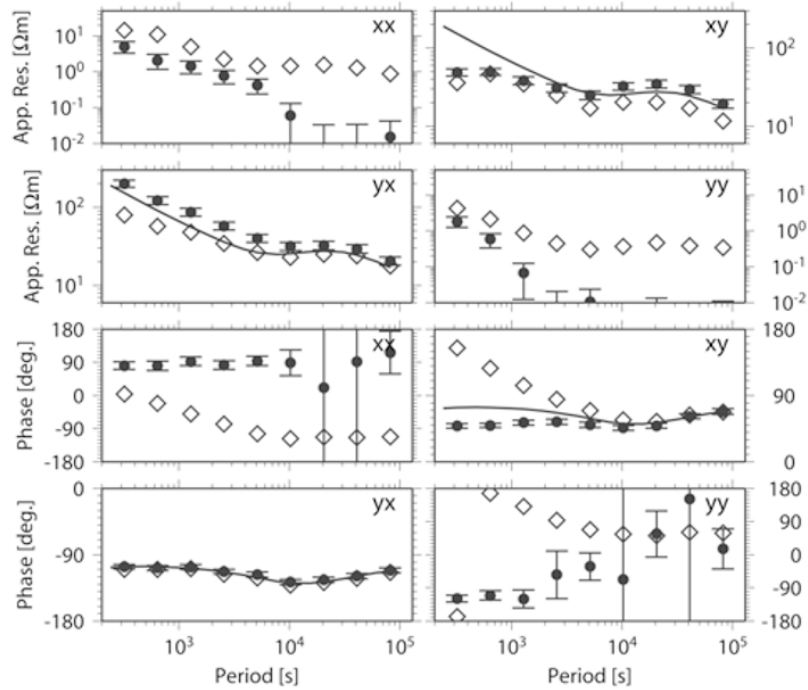

Fig. 2. Results using correction Eq. (1) (a), correction Eq. (2) (b), and correction Eq. (3) (c). Diamonds, dots, and solid lines denote synthetic, corrected, and flat-lying seafloor apparent resistivities and phases, respectively. Note that the scales between the diagonal and off-diagonal components are different. The error bars indicate $95 \%$ confidence limits and are shown only for the corrected apparent resistivity and phase.

$$
\mathbf{B}(\mathbf{r})=\mathbf{B}_{m}(\mathbf{r})+\mu_{0} \nabla \times \int_{V} \delta \frac{1}{\rho\left(\mathbf{r}^{\prime}\right)} \mathbf{G}\left(\mathbf{r}, \mathbf{r}^{\prime}\right) \mathbf{E}\left(\mathbf{r}^{\prime}\right) d \mathbf{r}^{\prime},
$$

where $\mathbf{r}$ is a position vector, $\mathbf{E}(\mathbf{r})$ is an electric field above an electrically anomalous body, $\delta\left(1 / \rho\left(\mathbf{r}^{\prime}\right)\right)$ is anomalous resistivity (or anomalous conductivity $\left.\delta \sigma\left(\mathbf{r}^{\prime}\right)\right), \mathbf{G}\left(\mathbf{r}, \mathbf{r}^{\prime}\right)$ is a Green's function, $V$ is the volume of the electrically anomalous body, $\omega$ is angular frequency, and $\mu_{0}$ is the permeability of free space. The approximations of Eqs. (5a) and (5b) lead to Eqs. (1a) and (1b). The topographic effect terms $\mathbf{M}$ and $\mathbf{K}_{e}$ depend on anomalous resistivity which is due to the existence of rugged seafloor topography and the resistivity contrast between seawater and the subsurface structure. To compare robustness of Eq. (1) to the assumed resistivity structure with that of Eq. (2), we rewrite Eq. (1b) using the fundamental equation of the magnetotelluric method $\mathbf{E}=\mathbf{Z B}$ as

$$
\mathbf{B}=\mathbf{B}_{m}+\mathbf{K}_{e} \mathbf{E}=\mathbf{B}_{m}+\mathbf{K}_{e} \mathbf{Z B} .
$$

Comparison of this equation with Eq. (2b) leads to the following equation,

$$
\mathbf{K}_{b}=\mathbf{K}_{e} \mathbf{Z}
$$

This equation indicates that $\mathbf{K}_{b}$ depends on $\mathbf{Z}$ which is the resistivity structure itself in addition to anomalous resistivity $\delta(1 / \rho)$. The correction with Eq. (2) is more directly influenced by the assumed resistivity structure than with Eq. (1). The topographic effect term $\mathbf{Z}_{t}$ in Eq. (3) also depends on $\mathbf{Z}_{m}$ which is the resistivity structure itself in addition to anomalous resistivity like as in Eq. (2). From Eqs. (1a) and (1b), the relationship between $\mathbf{Z}$ and $\mathbf{Z}_{m}$ is

$$
\mathbf{Z}=(\mathbf{I}-\mathbf{M})^{-1} \mathbf{Z}_{m}\left(\mathbf{I}-\mathbf{K}_{e} \mathbf{Z}\right),
$$

and therefore,

$$
\mathbf{Z}=\left(\mathbf{I}-\mathbf{M}+\mathbf{Z}_{m} \mathbf{K}_{e}\right)^{-1} \mathbf{Z}_{m} .
$$

Comparison of this equation with Eq. (3a) leads to the following equation

$$
\mathbf{Z}_{t}=\left(\mathbf{I}-\mathbf{M}+\mathbf{Z}_{m} \mathbf{K}_{e}\right)^{-1},
$$

This equation indicates that topographic effect term $\mathbf{Z}_{t}$ in Eq. (3) includes the resistivity structure itself $\mathbf{Z}_{m}$, suggesting that the correction with Eq. (3) is also more directly influenced by the assumed resistivity structure than with Eq. (1).

We investigated the dependence of the topographic correction on subsurface structures by assuming other two uniform resistivity structures. Resistivity values of the structures are $10 \mathrm{Ohm}-\mathrm{m}$ as a more conductive case compared to $100 \mathrm{Ohm}-\mathrm{m}$ uniform structure, and $1000 \mathrm{Ohm}-\mathrm{m}$ as a more resistive case. RMS misfits (Eq. (4)) are shown in Table 1. The RMS misfits with Eq. (1) are smaller than those with Eqs. (2) and (3) for any resistivities. This indicates that the dependence of the topographic correction on the subsurface structures with Eq. (1) is weaker than those with Eqs. (2) and (3), and that Eq. (1) is better at estimating unknown resistivity structures than Eqs. (2) and (3) when we estimate them from actual data observations. With Eqs. (2) and (3), the RMS misfits for the $100 \mathrm{Ohm}-\mathrm{m}$ uniform structure 
are smaller than those for the 10 and 1000 Ohm-m uniform structures. This is because the 100 Ohm-m uniform structure is closer to the true 1-D structure (Fig. 1(b)) than the other two uniform structures, indicating the high sensitivity of Eqs. (2) and (3) to the subsurface structures. On the other hand, the RMS misfits for the 100 and $1000 \mathrm{Ohm}$ m uniform structures with Eq. (1) are close to each other, indicating the high robustness of Eq. (1) to the higher resistive structure than the true 1-D structure. Incidentally, the RMS misfit for the $10 \mathrm{Ohm}-\mathrm{m}$ uniform structure with Eq. (1) is larger than those for the 100 and $1000 \mathrm{Ohm}-\mathrm{m}$ uniform structures, suggesting that the robustness of Eq. (1) is somewhat lower by assuming the lower resistive structure than the true 1-D structure. This lower robustness is supported by evidence that the topographic effect terms (M and $\mathbf{K}_{e}$ ) modeled for the $10 \mathrm{Ohm}-\mathrm{m}$ uniform structure deviate from those modeled for the 100 and 1000 Ohm-m uniform structures and for the true 1-D structure. Thus, the topographic correction with Eq. (1) shows higher robustness to subsurface structures compared to Eqs. (2) and (3). In addition, the topographic effect terms in Eq. (1) are modeled robustly by using a higher resistive structure than an anticipated structure.

\section{Topographic Correction of Real Data}

We applied the topographic correction with correction equation (1c) to real data observed in the Mariana area. The location of the observation site is the same as that of the synthetic data (Fig. 1(a)). The impedance tensor is computed from the time variations of the electric and magnetic fields which were recorded by an ocean bottom electromagnetometer for eight months (Seama et al., 2007). We correct the observed impedance tensor assuming a uniform half-space structure of $100 \mathrm{Ohm}-\mathrm{m}$ for calculating the topographic effect terms. Differences between the corrected (dots in Fig. 3) and the observed (diamonds in Fig. 3) responses exist in the xx component of the apparent resistivity at almost all the periods and in the other components of the apparent resistivity and phase at periods shorter than several thousands seconds. Since most discrepancies can be seen at these shorter periods, the impact of using correction equation (1) is obvious.

We estimated a smooth 1-D resistivity structure by using Occam's inversion (Constable et al., 1987), inverting the apparent resistivity and phase calculated from the square root of the determinant of the corrected impedance tensor $\left(\mathbf{Z}_{\mathrm{det}}\right) . \mathbf{Z}_{\mathrm{det}}$ is defined as

$$
\mathbf{Z}_{\mathrm{det}}=\sqrt{\mathbf{Z}_{x x} \mathbf{Z}_{y y}-\boldsymbol{Z}_{x y} \mathbf{Z}_{y x}}
$$

(e.g., Baba and Chave, 2005). The 1-D treatment is justified from $\rho+$ inversion (Parker and Booker, 1996) of $\mathbf{Z}_{\mathrm{det}}$ because the $\chi^{2}$ misfit of 19.5 is smaller than the $95 \%$ confidence limit of 51.0. In order to examine the validity of the estimated resistivity structure, the forward modeling with the estimated resistivity structure and seafloor topography is carried out, and the apparent resistivity and phase are calculated. The calculated (solid lines in Fig. 3) and observed responses show good agreement in almost all the components. The RMS misfit between the observed and calculated
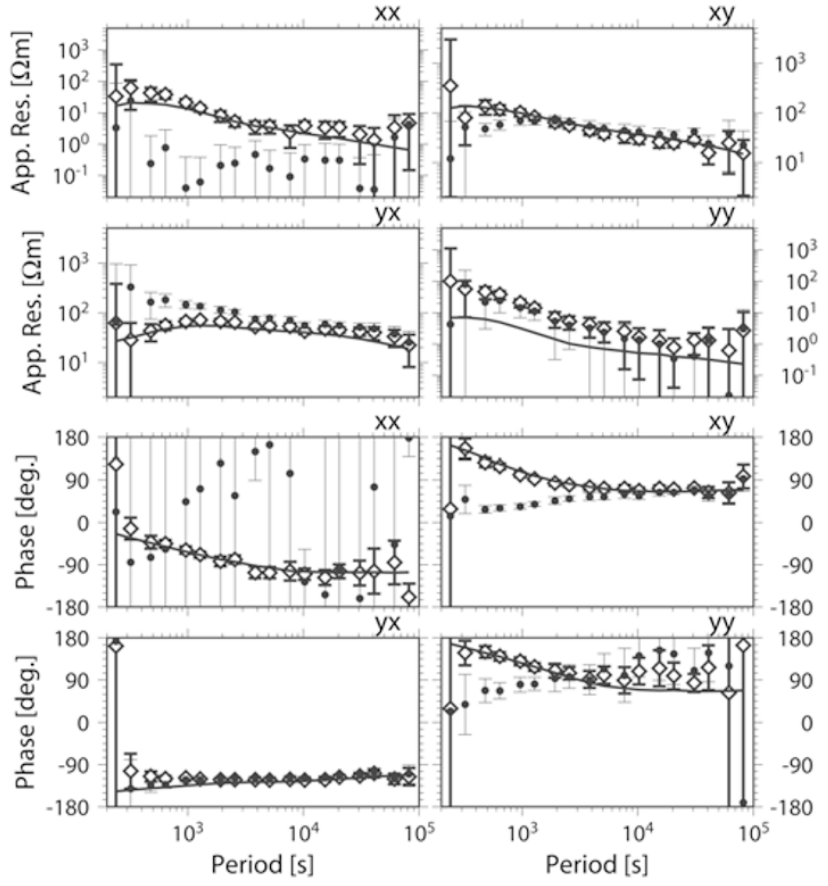

Fig. 3. Observed (diamonds), corrected (dots), and calculated (solid lines) apparent resistivities and phases. Note that the scales of the apparent resistivity are different between the diagonal and off-diagonal components. The error bars indicate $95 \%$ confidence limits.

responses is 2.7 , which is defined as

$$
\mathrm{RMS}=\sqrt{\frac{1}{2 N} \sum_{i=1}^{N}\left[\left\{\frac{\log \left(\rho_{o}^{i} / \rho^{i}\right)}{\delta \log \rho_{o}^{i}}\right\}^{2}+\left\{\frac{\phi_{o}^{i}-\phi^{i}}{\delta \phi_{o}^{i}}\right\}^{2}\right]},
$$

where $\rho$ is apparent resistivity, $\phi$ is phase, $\delta \log \rho$ and $\delta \phi$ are the standard errors of the apparent resistivity and phase, and $N$ is the number of data (Baba and Chave, 2005). This result indicates that the procedure to estimate the resistivity structure through the inversion after the topographic correction is effective.

\section{Conclusions}

The synthetic test using the three different topographic correction equations confirm that the topographic correction equation of Nolasco et al. (1998) is the best in application to distorted magnetotelluric data on the seafloor. The results of the synthetic test are most distinguishable at periods shorter than several thousands seconds. These differences are caused by the differences in treatment of the anomalous magnetic field and in robustness of the correction equation to the resistivity structure assumed for the correction. The anomalous horizontal magnetic field is explicitly proportional to the horizontal electric field in the equation of Nolasco et al. (1998). The equation of Nolasco et al. (1998) is more tolerant for a resistivity structure assumed for the correction and better accommodate the estimation of unknown resistivity structures. These results are recognized for the first time by using FS3D (Baba and Seama, 2002) which computes electromagnetic fields at shorter periods incorporating seafloor topography. The topographic correction and a 1-D inversion have been applied to real 
data observed in the Mariana area. The obtained resistivity structure explains almost all the components of the observed apparent resistivity and phase. We conclude that the inversion after the topographic correction by using the correction equation of Nolasco et al. (1998) and the forward modeling FS3D is a powerful procedure to estimate a reliable resistivity structure.

Acknowledgments. We appreciate critical and constructive comments by Pascal Tarits and two anonymous reviewers. We thank Antony White for improving the manuscript. T. M. also thanks the colleagues in Geodynamics Laboratory of Kobe University for their continuous encouragement and discussions. This research is supported by Grant-In-Aid for Scientific Research (B)(1) (No. 15340149), Japan Society for the Promotion of Science (JSPS), and "The 21st Century COE Program of Origin and Evolution of Planetary Systems" in Ministry of Education, Culture, Sports, Science, and Technology (MEXT). All the figures are created by using GMT software (Wessel and Smith, 1998).

\section{References}

Baba, K. and A. D. Chave, Correction of seafloor magnetotelluric data for topographic effects during inversion, J. Geophys. Res., 110, B12105, doi:10.1029/2004JB003463, 2005.

Baba, K. and N. Seama, A new technique for the incorporation of seafloor topography in electromagnetic modelling, Geophys. J. Int., 150, 392402, 2002.

Bahr, K., Interpretation of the magnetotelluric impedance tensor: regional induction and local telluric distortion, J. Geophys., 62, 119-127, 1988

Chave, A. D. and J. T. Smith, On electric and magnetic galvanic distortion tensor decompositions, J. Geophys. Res., 99(B3), 4669-4682, 1994.

Constable, S. and G. Heinson, Hawaiian hot-spot swell structure from seafloor MT sounding, Tectonophysics, 389, 111-124, 2004.

Constable, S. C., R. L. Parker, and C. G. Constable, Occam's inversion: A practical algorithm for generating smooth models from electromagetic sounding data, Geophysics, 52, 289-300, 1987.

Goto, T., T. Kasaya, H. Mikada, M. Kinoshita, K. Suyehiro, T. Kimura, Y. Ashida, T. Watanabe, and K. Yamane, Electromagnetic survey of fluid distribution and migration — an example at the Nankai seismogenic zone, BUTSURI-TANSA, 56, 439-451, 2003 (in Japanese with English abstract).

Groom, R. W. and R. C. Bailey, Decomposition of magnetotelluric impedance tensors in the presence local three-dimensional galvanic distortion, J. Geophys. Res., 94(B2), 1913-1925, 1989.

Heinson, G. S. and F. E. M. Lilley, An application of thin-sheet electromagnetic modelling to the Tasman Sea, Phys. Earth Planet. Inter., 81, 231-251, 1993.

Hohmann, G. W., Three-dimensional induced polarization and electromagnetic modeling, Geophysics, 40, 309-324, 1975.

Jiracek, G. R., Near-surface and topographic distortions in electromagnetic induction, Surv. Geophys., 11, 163-203, 1990.

Jiracek, G. R., R. P. Reddig, and R. K. Kojima, Application of the Rayleigh-FFT technique to magnetotelluric modeling and correction, Phys. Earth Planet. Inter., 53, 365-375, 1989.

Mackie, R. L., J. T. Smith, and T. R. Madden, Three-dimensional electromagnetic modeling using finite difference equations: The magnetotelluric example, Radio Science, 29, 923-935, 1994.

Nolasco, R., P. Tarits, J. H. Filloux, and A. D. Chave, Magnetotelluric imaging of the Society Island hotspot, J. Geophys. Res., 103(B12), 30,287-30,309, 1998.

Parker, R. L. and J. R. Booker, Optimal one-dimensional inversion and bounding of magnetotelluric apparent resistivity and phase measurements, Phys. Earth Planet. Inter., 98, 269-282, 1996.

Schmucker, U., Anomalies of geomagnetic variations in the Southwestern United States, Bull. Scripps Inst. Oceanogr., Univ. Calif. Press, 13, 165, 1970.

Schwalenberg, K. and R. N. Edwards, The effect of seafloor topography on magnetotelluric fields: an analytical formulation confirmed with numerical result, Geophys. J. Int., 159, 607-621, 2004.

Seama, N., K. Baba, H. Utada, H. Toh, N. Tada, M. Ichiki, and T. Matsuno, 1-D electrical conductivity structure beneath the Philippine Sea: Results from an ocean bottom magnetotelluric survey, Phys. Earth Planet. Inter., 162, 2-12, doi:10.1016/j.pepi.2007.02.014, 2007.

Vasseur, G. and P. Weidelt, Bimodal electromagnetic induction in nonuniform thin sheets with an application to the northern Pyrenean induction anomaly, Geophys. J. R. astr. Soc., 51, 669-690, 1977.

Wessel, P. and W. H. Smith, New, improved version of Generic Mapping Tools released, EOS Trans. AGU, 79, 579, 1998.

T. Matsuno (e-mail: matsuno@kobe-u.ac.jp), N. Seama, and K. Baba 